\title{
Oral Health Impact Profile in Orthodontic patient with Ectopic canine: A prospective clinical intervention of four treatment modalities
}

Mohammad Khursheed Alam ( $\nabla$ mkalam@ju.edu.sa )

Associate Professor, Orthodontic Division, HOD, Preventive Dentistry Department, College of Dentistry, Jouf University. Sakaka, Al Jouf, KSA. https://orcid.org/0000-0001-7131-1752

\section{Research article}

Keywords: OHRQoL; OHIP-16; ectopic canine; orthodontic treatment modalities

Posted Date: May 31st, 2019

DOI: https://doi.org/10.21203/rs.2.9958/v1

License: (9) This work is licensed under a Creative Commons Attribution 4.0 International License. Read Full License

Version of Record: A version of this preprint was published at Healthcare on March 16th, 2021. See the published version at https://doi.org/10.3390/healthcare9030337. 


\section{Abstract}

Objective To evaluate oral health related quality of life (OHRQoL) in Saudi subjects with ectopic canine, severe crowding and Class I normal occlusion and to assess the differences among different orthodontic treatment modalities [OTM] for baseline, 1-day and 28-day after fixed orthodontic treatment. Materials and Methods The study sample comprised 32 subjects with ectopic canine who were attending orthodontic clinics at the college of Dentistry. Subjects were equally grouped in to 4 based on OTM. Each participant was assessed for OHRQoL using the Oral Health Impact Profile, English version (OHIP-16). Initial comparison of baseline data of ectopic canine subjects was done with severe crowding $(>8 \mathrm{~mm})$ and Class I normal occlusion with the matched number and aged subjects in later groups. Also, comparison of OHIP-16 scores among 4 OTM, baseline, 1-day and 28-day after treatment were done. Results OHIP-16 scores of ectopic canine, severe crowding and Class I normal occlusion group was $33.06,33.09$ and 26.43 respectively. Subjects with ectopic canine and severe crowding reported a significantly greater negative impact on the embarrassment, avoid smiling and lack of self-confidence however, overall OHRQoL score was insignificant. OHRQoL (total OHIP-16 score and score for each domain) have no significant changes among 4 OTM group $(P>0.05)$. Significant dissimilarities in OHIP16 scores were apparent with respect to treatment time. Conclusion Untreated malocclusion has a significant negative impact on OHRQoL. The greatest negative impact was seen in the several domain 1day after treatment however, gradual improvement noted 28-day after treatment irrespective of 4 different OTM.

\section{Introduction}

Correction of malposed teeth and discrepancies related to the jaw growth and their relation is being done by orthodontics since its inception. Such treatment has positive effect on functional demands, dental health and aesthetics. It also has significant effect on the community, corporeal and psychological comfort (Fernandes et al., 2001; Javidi et al., 2017). ${ }^{1-2}$ However, fixed orthodontic treatment is also associated with deleterious effects which have an impact on the quality of life. Hence, a significant drive for assessment of oral health related quality of life (OHRQoL) in orthodontics is gaining momentum world over ( Liu et al. 2009; Zubir et al. 2017) . 3-4

Every tooth, as it erupts from its developmental position within the jaws to its functional position in the occlusal plane, it erupts into a specific, predetermined position in the dental arch. However, sometimes there is a deviation in its path of eruption and such teeth erupt in abnormal locations which are termed as ectopic eruption. According to Nikiforuk (1948) ${ }^{5}$, ectopic eruption is a condition where the permanent tooth takes an abnormal path of eruption due to deficiency in the jaw growth or a segment of jaw and encounters a deciduous tooth leading to its premature exfoliation and malposition of the permanent tooth (Nikiforuk, 1948). ${ }^{5}$

The maxillary and mandibular canines are anterior teeth which are placed at the corners of the mouth and their positions and forms of these teeth along with the canine eminence play a vital role in aesthetics 
and face value. The inclination of the upper canine also influences the aesthetics of a smile (Bothung et al. 2015). ${ }^{6}$ Hence, any change in its position or early loss may not only unduly affect the general appearance but may also psychologically impact an individual.

The underlying concepts of various OTM in therapeutic orthodontics are simple and easily understood. However, the type, degree of malocclusion, treatment stages and the choice of treatment modality may affect the treatment duration. It can be assumed that the impact of ectopically placed canine can affect the psychological development of the child/individual and may in general affect the quality of life. Early diagnosis of such ectopically developing maxillary canines and instituting the corrective measures would minimize the negative impact of teeth and boost up the morale and confidence of the individual affected.

Studies revealed, low level laser therapy (LLLT) has promising benefits on osteogenesis in-vitro (Jawad et al. 20013, 2018), ${ }^{7-8}$ on bone remodelling during orthodontic tooth movement in rats (Jawad et al. 2018), ${ }^{9}$ on acceleration of orthodontic tooth movement in animal (Qamruddin et al. 2015) ${ }^{10}$ and human (Qamruddin et al. 2017) ${ }^{11}$, on spontaneous and chewing pain caused by elastomeric separators (Qamruddin et al. 2016) ${ }^{12}$, on pain associated with the initial stage of fixed orthodontic treatment (Qamruddin et al. 2018)..$^{13}$ However, this TM has never been investigated on OHRQoL.

This first-in-human study compared OHRQoL using OHIP-16 scores among ectopic canine, severe crowding and Class I normal occlusion subjects' group and the changes in OHRQoL using OHIP-16 scores (Mansor et al. 2012) ${ }^{14}$, baseline, 1-day, and 28-day following insertion of fixed orthodontic appliances in 4 different TM groups in ectopic canine subjects.

\section{Material And Methods}

Thirty-two healthy orthodontic patients of Saudi ethnic background with ages between 14 and 25 years were selected for the study. Based on TM all patients are randomly divided into 4 groups, these are LLLT+ (self-ligating bracket) SLB, non-LLLT+SLB, LLLT+ conventional bracket (Conv.) and non-LLLT+Conv. group. Inclusion, exclusion criteria, study groups, 4 different TM, number of subjects in each group, armamentarium used, orthodontic treatment, laser application, OHIP-16 assessment (Mansor et al. 2012) ${ }^{14}$, ethical aspects and statistical analysis are detailed in Table 1. Figure 1 show the conceptual framework the current study.

For initial comparison with the study subjects, matched with age, and number, severe crowding and Class I normal occlusion subjects OHIP-16 data were collected. OHIP-16 score among 3 groups (ectopic canine, severe crowding [ $>8 \mathrm{~mm}$ crowing] and Class I normal occlusion [Class I molar, incisor and canine relation, without any crowding, normal overjet and overbite, without any orthodontic treatment]) were analysed using one way ANOVA to see the differences.

\section{Results}


Among 7 domains of OHIP-16 scores, psychological discomfort (Embarrassment, $p=>0.001$ ), physical disability (Avoid smiling, $p=>0.001$ ) and Handicap (Lack of self-confidence, $p=>0.001$ ) showed highly significant difference among 3 groups (Table 2). Total OHIP-16 scores of ectopic canines and severe crowding subjects' group was comparatively higher then Class I normal occlusion group, however no significant differences were found among 3 groups (Table 2).

Table 3 shows Mean \pm SD values of all 7 domains of OHIP-16 scores among the 4 different TM groups for base line, 1-day and 28-day after fixed orthodontics treatment.

Figure 2 shows the factor plot charts of OHIP-16 scores of 4 different TM groups base line, 1-day and 28day after fixed orthodontics treatment. No significant differences were in all OHIP-16 scores among 4 TM groups. However, treatment time duration showed significant changes in all OHIP-16 scores.

\section{Discussion}

The patients with orthodontic needs approach a clinician primarily to address their dentofacial aesthetics and improve their oral health (Ackermann, 2007).${ }^{15}$ However, like in other interventions, treatment with fixed orthodontic appliances is also not free of associated complications or risks. Pain, mobility, delayed tooth movement, root resorption and loss of vitality at times, may be some of the associated untoward effects of the treatment which could be due to excessive force applied for orthodontic tooth movement ( Talic, 2011). ${ }^{16}$ In addition, due to difficulty in brushing there may be accumulation of plaque around the brackets resulting in halitosis, decalcification of tooth surface, gingival inflammation and periodontal disease ( Lau and Wong, 2006). ${ }^{17}$

Previous studies relating to malocclusion and orthodontic treatment with OHRQoL are found to ambiguous. While few studies proved that there is correlation orthodontic need or malocclusion and OHRQoL, few others indicated that there is no clear relationship (Andiappan et al. 2014; Herabiet al. 2011). ${ }^{18-19}$ In this study, statistical analysis showed that participants with severe malocclusions reported significantly greater negative impact on OHRQoL (Table 2). The varied findings of these studies could be due to differences in the use of measures, age groups, cultures, traditions, races \& ethnic groups and social norms across countries.

In this study, embarrassment, avoid smiling and lack of self-confidence was significantly higher in severe crowding group followed by ectopic canine compared to Class I normal occlusion group. Our findings suggest that the patients suffered from aesthetic and social issues. However, their daily activities remained unaffected and that any small variations in orthodontic treatment needs might significantly affect perceived OHRQoL in the psychological discomfort domain. Studies by Oliveira et al., (2004) ${ }^{20}$, $\mathrm{O}^{\prime}$ Brien et al., (2007) $)^{21}$ and Feu et al., (2013) ${ }^{22}$ strongly suggest that there is a positive association between malocclusion and psychological discomfort. ${ }^{20-22}$ Questions relating to emotional and social domains including getting upset, embarrassment, shyness and stop smiling or laughing in the psychological scales are more applicable to orthodontic patients (de Paula et al. 2009). ${ }^{23}$ Secondly, the 
patients with greater orthodontic needs presented greater psychological impairment as compared to those with no or borderline treatment needs. According to Zhou et al. (2001) ${ }^{24}$ about of half of the patients with malposed teeth had nicknames associated with their dentofacial problems and that $80 \%$ of these patients were angry or upset about their nickname. Also, it was shown by Zhou et al $(2001)^{24}$ that the psychological status of patients with skeletal malocclusion was directly related to the severity of malocclusion.

Patients with malocclusion may get easily embarrassed in social contexts and their self-concept may be negatively affected (de Paula et al. 2009; Zhang et al. 2006). ${ }^{23,25}$ Most of the patients seeking orthodontic treatment want their dental aesthetics corrected and improve self-esteem (Feu et al., 2013). ${ }^{22}$ Thus, it is important that the orthodontist plans the treatment to improve oral functioning and health, improve aesthetics, self-esteem and social activities. As a part of diagnostic procedure, collecting data pertaining to OHRQoL may indicate the priorities for treatment to achieve maximum patient satisfaction (Feu et al. 2013). ${ }^{22}$ However, the OHIP-16 has only four items (of 16) related to evaluation of psychological status. Liu et al $(2011)^{26}$ have suggested that an ideal instrument with more considerations of psychological aspects for assessing orthodontic related quality of life research may be required as it may paly significant role in evaluating quality of life in patients with malocclusion.

Irrespective of the reason, adverse effects of treatment proportionate directly with the duration of treatment. Currently the duration of orthodontic treatment with fixed braces is 2 to 3 years on average ( Fink and Smith, 1992). ${ }^{27}$ However the patient does not expect more than 1.5 years ( Sayers and Newton, 2007). ${ }^{28}$ Prolonged treatment duration may also be deleterious efficiency of national health care system and also private practices. Thus, reducing the duration of the treatment by accelerating the tooth movement has been perennial concern for both orthodontists and patients (Jawad et al. 2015). ${ }^{29}$ OHRQoL in orthodontic patients essentially depends on these variables these variables and outcome. Current research explored the OHRQoL based on the 4 different TM, laser and non-laser in CB and SLB were observed for baseline, 1-day and 28-day after fixed orthodontic treatment. Consequently, no significant differences were observed. Thus, all modalities can enhance the quality of life. These 4 TM has its pros and cons but in regard to OHRQoL, all have beneficial effect. Current research investigated the OHIP-16 scores in stage wise treatment. As per finding the OHRQoL, 1-day and 28-day after orthodontic treatment regardless of different TM has been changed. Drastic changes in all 7 domains of OHIP-16 scores are revealed 1-day after orthodontic treatment. These findings are quite similar to Mansor et al. (2012) $)^{14}$ in relation to 1-day after orthodontic treatment. Other follow up comparisons are not possible because of the study was concluded with the 1-day after orthodontic treatment data only. OHIP16 scores for baseline, 1-day and 28-day after fixed orthodontic treatments of 4 different TM, almost no changes in OHRQoL in domain number 2 (Physical pain), 5 (Psychological disability) and 6 (Social disability). Among OHIP-16, question 1 (Difficulties in chewing), 4 (Discomfort in eating), 7 (Food stuck in between teeth) and 9 (Avoidance of eating certain foods) showed bad impact in OHRQoL 1-day after orthodontic treatment. However, the bad impact gradually reduces 28-day after orthodontic treatment. Furthermore, domain number 3 (Psychological discomfort) question 8 (Embarrassment) and domain 
number 4 (Physical disability) question 10 (Avoid smiling) showed good impact 28-day after orthodontic treatment.

This first-in-human study revealed some significant and non-significant results. Three different participant group comparison on OHRQoL has been done. Four different OTM groups with 3 different level data comparison also assessed in ectopic canine group. However, there are some limitation needed to be overcome to reveal confirmatory results. Longitudinal follow-up with larger sample size is important. This study has been done in a single ethnic population, future studies with different population might reveal some disparities in OHRQoL. Researcher encourages to do similar type of study in different subject group, different OTM and in different population.

Conclusion: This first-in-human research explored the OHRQoL in ectopic canine, severe crowding and Class I normal occlusion subjects. Highly significant disparities were revealed among 3 groups. This study also revealed, among 4 different TM group in ectopic canine subjects have no significant OHIP-16 scores. For the base line, 1-day and 28-day after fixed orthodontics treatment OHRQOL were significantly different.

\section{List Of Abbreviations}

OHIP - Oral health impact profile

OHRQOL - Oral health related quality of life

OTM - Orthodontic tooth movement

TM - Treatment modalities

LLLT - Low level laser therapy

SLB - Self ligating bracket

Conv. - Conventional

\section{Declarations}

Compliance with Ethical Standards

* Funding - This study was funded by research fund\#Jouf University\#40/001

* Conflict of Interest: Author declares that he has no conflict of interest.

* Ethical approval - Approval obtained from the Ethical Committee of the Jouf University [LCBE\#4-222/40], which complies with the Declaration of Helsinki. Once patients have agreed to participate in the study, researcher explained about the study to the patients and the nature of their participation. 
* Research involving human participants - Each patient was given an oral and a written information sheet about the study and written informed consent was obtained before the first questionnaire was administered. For patients below 18 years old, consent was obtained from the patients and parents. All patients signed informed consent forms that described the purpose, benefits, and drawbacks of the study.

Availability of data and material: Within the manuscript in the form of text in the result and tables and figures.

Competing interests: None declared

Authors' contributions: Lead author and correspondence. Conception, design, research, data collection, data analysis, results, discussion, manuscript draft, final editing and submission.

\section{References}

1. Fernandes MJ, Ruta DA, Ogden GR, Pitts NB, Ogston SA. Assessing oral health-related quality of life in general dental practice in Scotland: validation of the OHIP-14. Community Dent Oral Epidemio 2006;34(1):53-62.

2. Javidi $\mathrm{H}$, Vettore $\mathrm{M}$, Benson PE. Does orthodontic treatment before the age of 18 years improve oral health-related quality of life? A systematic review and meta-analysis. Am J Orthod Dentofac Orthoped 2017;151(4):644-655.

3. Liu Z, McGrath C, Hägg U. The impact of malocclusion/orthodontic treatment need on the quality of life: a systematic review. Angle Orthod 2009;79(3):585-591.

4. Zubir ZM, Masood M, Rahman ANAA. An Assessment of Oral Health Related Quality of Life in Orthodontics Patients with Micro-Implant: A Pilot Study. UI Proceedings Health Med 2017;1;131-135.

5. Nikiforuk G. Ectopic Eruption: Discussion and clinical report. J Ont Dent Assoc 1948;25:243-6.

6. Bothung C, Fischer K, Schiffer, Springer I, Wolfart S. Upper canine inclination influences the aesthetics of a smile. J Oral Rehabil 2015;42(2):144-52.

7. Jawad MM, Husein A, Azlina A, Alam MK, Hassan R, Shaari R. Effect of 940nm low level laser therapy on osteogenesis in vitro. J Biomed Optics 2013;18(12):128001.

8. Jawad MM, Husein A, Azlina A, Alam MK, Hassan R, Shaari R. Effects of low level laser therapy and low intensity pulsed ultrasound treatment and the combination of them on osteogenesis in vitro. Int $\mathrm{J}$ Orthod 2018;29(2):60-67.

9. Jawad MM, Husein A, Alam MK, Hassan R, Shaari R, Azlina A, Salzihan MS. Effect of low level laser and low intensity pulsed ultrasound therapy on bone remodeling during orthodontic tooth movement in rats. Progress Orthod 2018;19:10. 
10. Qamruddin I, Alam MK, Khamis MF, Husein A. Minimally Invasive Techniques to Accelerate the Orthodontic Tooth Movement: A Systematic Review of Animal Studies. BioMed Research Int. 2015; Article ID 608530, 10 pages.

11. Qamruddin I, Alam MK, Fida M, Khan AG. Effect of a single dose of low-level laser therapy on spontaneous and chewing pain caused by elastomeric separators. Am J Orthod Dentofacial Orthop 2016;149(1):62-66.

12. Qamruddin I, Alam MK, Mahroof V, Fida M, Khamis MF, Husein A. Effects of low-level laser irradiation on the rate of orthodontic tooth movement and associated pain with self-ligating brackets. Am J Orthod Dentofacial Orthop 2017;152:622-30.

13. Qamruddin I, Alam MK, Abdullah H, Kamran MA, Jawaid N, Mahroof V. Effects of single-dose, lowlevel laser therapy on pain associated with the initial stage of fixed orthodontic treatment: A randomized clinical trial. Korean J Orthod 2018;48(2):90-97.

14. Mansor N, Saub R, Othman SA. Changes in the oral health-related quality of life $24 \mathrm{~h}$ following insertion of fixed orthodontic appliances. J Orthodont Sci 2012;1:98-102.

15. Ackerman MB. Enhancement orthodontics: Theory and practice: Wiley-Blackwell. 2007.

16. Talic NF. Adverse effects of orthodontic treatment: a clinical perspective. Saudi Dent J 2011;23(2):5559.

17. Lau PY-W, Wong RW-K. Risks and complications in orthodontic treatment. 2006.

18. Andiappan M, Gao W, Bernabé E, Kandala NB, Donaldson AN. Malocclusion, orthodontic treatment, and the Oral Health Impact Profile (OHIP-14) systematic review and meta-analysis. Angle Orthod 2015;85(3):493-500.

19. Heravi F, Farzanegan F, Tabatabaee M, Sadeghi M. Do malocclusions affect the oral health-related quality of life? Oral Health Prev Dent 2011;9:229-233.

20. de Oliveira $\mathrm{CM}$, Sheiham A. Orthodontic treatment and its impact on oral health-related quality of life in Brazilian adolescents. J Orthod 2004;31:20-27.

21. O'Brien C, Benson PE, Marshman Z. Evaluation of a quality of life measure for children with malocclusion. J Orthod 2007;34:185-193, 176. 22.

22. Feu D, Miguel JA, Celeste RK, Oliveira BH. Effect of orthodontic treatment on oral health-related quality of life. Angle Orthod 2013;83:892-898.

23. de Paula JD, Santos NC, da Silva ET, Nunes MF, Leles CR. Psychosocial impact of dental esthetics on quality of life in adolescents. Angle Orthod 2009;79:1188-1193. 
24. Zhou YH, Hagg U, Rabie AB. Concerns and motivations of skeletal Class III patients receiving orthodontic-surgical correction. Int J Adult Orthodon Orthognath Surg 2001;16: 7-17.

25. Zhang M, McGrath C, Ha"gg U. The impact of malocclusion and its treatment on quality of life: a literature review. Int J Paediatr Dent 2006;16:381-387.

26. Liu Z, McGrath C, Ha“gg U. Associations between orthodontic treatment need and oral health-related quality of life among young adults: does it depend on how you assess them? Community Dent Oral Epidemiol 2011;39:137-144.

27. Fink DF, Smith RJ. The duration of orthodontic treatment. Am J Orthod Dentofac Orthoped 1992;102(1):45-51.

28. Sayers M, Newton J. Patients' expectations of orthodontic treatment: Part 2-findings from a questionnaire survey. J Orthod 2007;34(1):25-35.

29. Jawad MM, A Husein, MK Alam, R Hassan, R Shaari. Overview of non-invasive Factors (low level laser and low intensity pulsed ultrasound) accelerating tooth movement during orthodontic treatment. Lasers Med Sci 2015;29(1):367-72.

\section{Tables}

Due to technical limitations, all Tables are only available as a download in the supplemental files section.

\section{Figures}




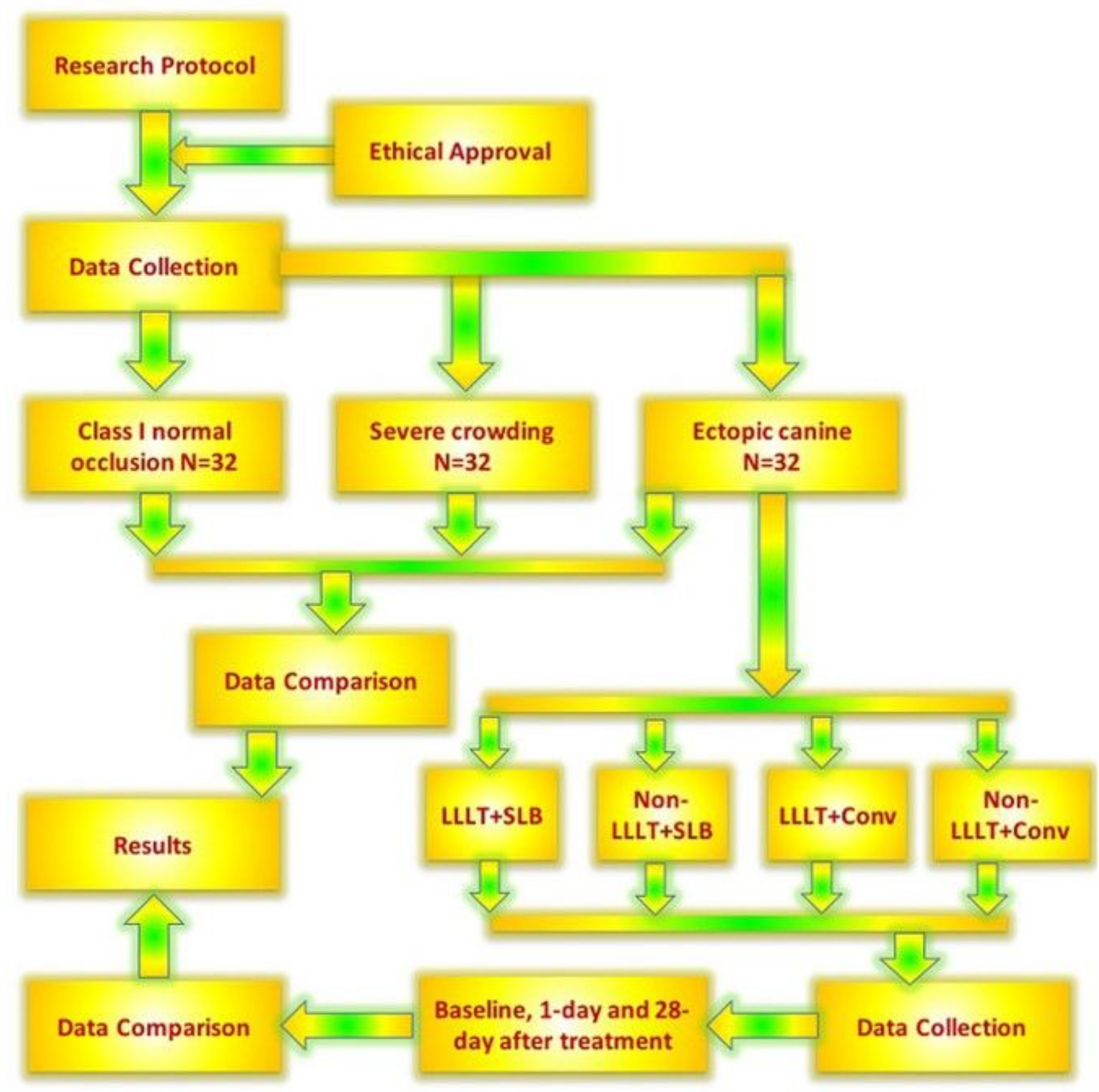

Figure 1

Conceptual framework of the research 

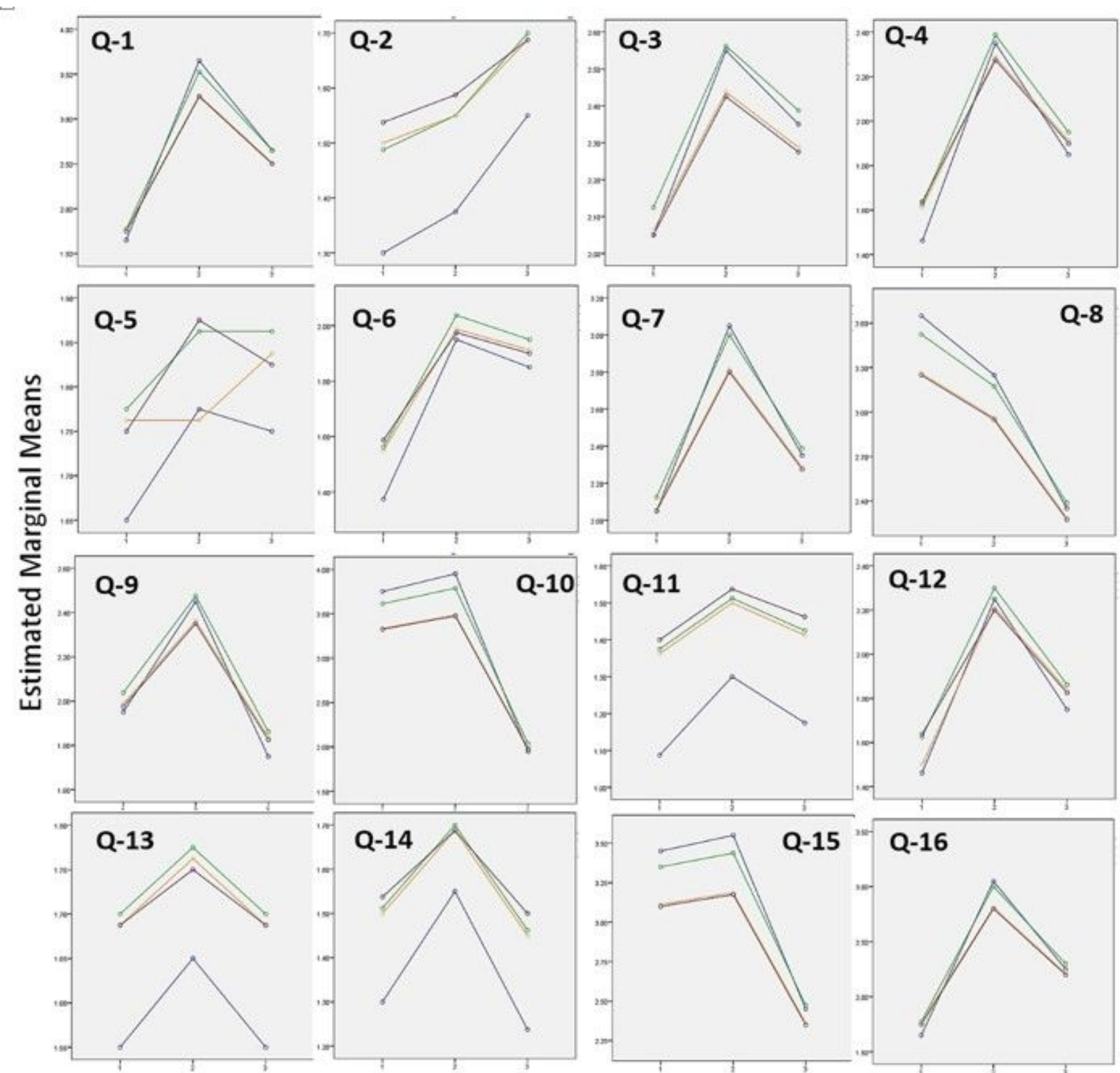

Treatment Time

Conv $+\mathrm{NL}$

Conv $+\mathrm{I}$

$\mathrm{SL}+\mathrm{NI}$

$\mathrm{SL}+$

Treatment time: $1=$ Baseline, $2=1$-day after bonding and $3=28$-days after bonding.

\section{Figure 2}

Factor plot charts of OHIP-16 scores of 4 different TM groups

\section{Supplementary Files}

This is a list of supplementary files associated with this preprint. Click to download. 
- Tables.docx

Page $12 / 12$ 